\title{
Análise do processo de gestão do conhecimento em uma empresa de base tecnológica da cidade de Joinville-SC
}

\begin{abstract}
RESUMO
Atualmente o conhecimento é considerado o principal fator de produção das organizações. Dentre as empresas que mais se destacam como intensivas em conhecimento, estão as organizações de base tecnológica. Assim, o objetivo do presente estudo é mostrar as ações de gestão do conhecimento realizadas por uma empresa de base tecnológica de Joinville/SC. Foi realizado um estudo de caso qualitativo, de caráter exploratório e explicativo, com utilização de dados primários, coletados em duas etapas. A primeira coleta foi feita por meio de um instrumento de pesquisa com perguntas abertas, enviadas por e-mail, a dois colaboradores da organização em foco; e a segunda, por intermédio de ligações telefônicas para os mesmos colaboradores com o objetivo de conhecer melhor a realidade da empresa e analisar as respostas dadas ao questionário. Dentre os principais resultados encontrados, pode-se citar o fato de a empresa fazer uso de ferramentas como Comunidades de Prática, identificadas na rotina organizacional.
\end{abstract}

Palavras-chave: Gestão. Conhecimento. Empresa. Tecnologia.

Evelin Priscila Trindade evelin.trindade@gmail.com Mestranda em Engenharia e Gestão do Conhecimento da UFSC

Marilei Osinski marileiosinski@gmail.com Mestranda em Engenharia e Gestão do Conhecimento da UFSC

Fernando Alvaro Ostuni Gauthier fernando.gauthier@ufs.br Universidade Federal de Santa Catarina

Paulo Mauricio Selig selig@egc.ufsc.br Universidade Federal de Santa Catarina

Marcelo Macedo 


\section{INTRODUÇÃO}

Lobo (2010) ressalta que o conhecimento transformou-se no agente fundamental no mundo dos negócios e tornou-se um recurso necessário em todos os processos organizacionais que envolvam o homem. De acordo com Uriarte Jr. (2008), não existe uma definição aceita universalmente de gestão do conhecimento. Mas, pode-se considerar a gestão do conhecimento como um processo pelo qual organizações geram valor com base em seus ativos intelectuais e base de conhecimento.

Nesse contexto, as organizações de base tecnológica são as que mais se destacam. As empresas de base tecnológica, como a escolhida para este trabalho, são firmas cuja intensa dinâmica inovadora sustenta-se em sólidas competências técnicas, havendo em relação a elas uma expectativa de crescimento acelerado (SANTOS; PINHO, 2010). Para um estudo mais aprofundado da aplicação da gestão do conhecimento efetivamente, é importante conhecer as ações de gestão do conhecimento adotadas por uma organização de base tecnológica. Portanto, este texto tem como objetivo principal mostrar as ações de gestão do conhecimento realizadas por uma empresa de base tecnológica de Joinville/SC.

A presente pesquisa é um estudo de caso, em que dois colaboradores da empresa responderam, inicialmente, a um questionário aberto e, posteriormente, às respostas por meio de contato telefônico. A fim de compreender o processo de gestão do conhecimento da empresa, os colaboradores entrevistados foram o gestor do conhecimento e um analista de software, que é usuário do conhecimento da empresa.

O artigo é organizado com as seguintes seções: introdução, gestão do conhecimento, compartilhamento do conhecimento organizacional, gestão do conhecimento em empresas de base tecnológica, procedimentos metodológicos, análise dos resultados, considerações finais e referências.

\section{GESTÃO DO CONHECIMENTO}

A sociedade é transformada, tanto nos aspectos econômicos quanto sociais, pela evolução do conhecimento. As pessoas e as organizações passaram a compreender que um diferencial, além do conhecimento em si, é a forma como este é compartilhado no ambiente organizacional, para promover a inovação de produtos e/ou serviços. Tal compreensão pode ter sido causada pelo aumento da competitividade, que exige mais eficiência e rapidez na gestão do conhecimento organizacional (FREIRE et al., 2012).

Autores como Sá et al. (2013) destacam que, por causa da competitividade organizacional existente, os ativos intangíveis estão sendo percebidos, de forma crescente, como possibilidade de criação de novos valores. Nesse sentido, a gestão do conhecimento recebe cada vez mais atenção, especialmente por parte das empresas que buscam formas de gestão capazes de proporcionar impactos positivos no desempenho organizacional.

A gestão do conhecimento promove às organizações a criação, conversão e uso apropriado do conhecimento, beneficiando os negócios. Além disso, procura identificar as formas mais eficientes de mobilizar conhecimentos individuais, buscando torná-los coletivos e integrá-los a estratégias que resultem em vantagens competitivas. Fazem parte desse processo práticas e procedimentos que se dedicam a criar competências em todos os níveis organizacionais (JACQUES; GONÇALO, 2007).

De acordo com Freire et al. (2012), todas as organizações utilizam e geram conhecimentos no decorrer de seus processos, consistindo num conjunto de tarefas específicas, desenvolvidas no meio organizacional. À medida que as organizações detêm mais conhecimento (a respeito do mercado, seus produtos, processos, clientes, tecnologia, concorrentes, entre outros fatores), ou administram eficientemente a aquisição, disseminação e preservação do conhecimento, tendem a ser bemsucedidas. Para que isso seja possível, os autores ressaltam que é necessário que o processo de construção do conhecimento organizacional seja administrado.

Segundo Pereira et al. (2009), as organizações estão inseridas em um ambiente dinâmico, com competitividade crescente e mudanças radicais e contínuas, que supostamente não são enfrentadas desde a Revolução Industrial. Nesse contexto, o conhecimento pode ser considerado a nova moeda de troca, pois novos paradigmas são quebrados e/ou surgem constantemente, exigindo abordagens modernas tanto pessoais quanto profissionais.

Hoje em dia, as empresas precisam, constantemente, buscar um diferencial competitivo para fortalecê-las e mantê-las no mercado. Com esse propósito, adotar uma forma de gestão do conhecimento por meio da qual os conhecimentos presentes nas empresas podem ser organizados em prol destas pode trazer pontos positivos à realidade organizacional. Por outro lado, caso a 
mensagem não seja bem passada, os colaboradores podem não entender a sua importância e pensar que partilhar seus conhecimentos significa perder a propriedade intelectual das próprias ideias, assim como dos métodos e dos processos utilizados (SÁ et al., 2013).

Nesse contexto, as organizações devem administrar o conhecimento do seu capital/ativo intelectual, buscando não apenas manter, mas também ampliar vantagens competitivas perante seus concorrentes. Em outras palavras, as organizações devem utilizar ferramentas da gestão do conhecimento a fim de criar novos conhecimentos, com base na renovação e atualização das informações, bem como com a utilização da sua memória organizacional. Nesse ambiente, o conhecimento - envolvendo a gestão dos valores intangíveis da organização, além dos tangíveis - tornou-se um dos fatores mais importantes para a competitividade organizacional (FREIRE et al., 2012).

A globalização está submetida ao conhecimento, competência individual e surgimento de competidores cada vez mais empreendedores e competitivos. Diante desse ambiente turbulento, o conhecimento passa a ser o caminho em que sistemas, ideias e pessoas convertem-se em valor agregado. Saber gerenciar o conhecimento tornou-se regra geral para as organizações inseridas no contexto atual e colocá-lo a serviço da geração de valor mais rapidamente que a concorrência, e torná-lo um processo constante é uma das alternativas de sobrevivência das empresas diante da atual configuração da sociedade (PEREIRA et al., 2009).

Nesse sentido, Freire et al. (2012) explicam que o ambiente organizacional deve ser organizado e gerenciado de modo a permitir a passagem da informação para conhecimento, assim como para que ocorra o compartilhamento de tarefas de trabalho, experiências e habilidades pessoais (conhecimento tácito) para o grupo. Além disso, o conhecimento deve ser explicitado e codificado (tornando-se assim conhecimento explícito) para ser armazenado e utilizado por outras pessoas na criação de novos conhecimentos.

Segundo Evers (2001), a próxima transformação no pensamento gerencial utiliza o conhecimento e a informação para descobrir e atender a necessidades sociais ainda não providas. Ao atender a tais necessidades e solucionar problemas, percebe-se uma mudança na sociedade, que evolui de uma sociedade industrial para a sociedade do conhecimento. De acordo com Pereira et al. (2009), tal transição de uma sociedade industrial para uma sociedade baseada no conhecimento e na informação trouxe mudanças significativas para o ambiente organizacional e social.

$\mathrm{Na}$ nova sociedade, a do conhecimento, é necessário haver um nível de escolaridade mais elevado do que nos demais tipos de sociedade existentes. Nessa nova sociedade, as organizações são transformadas em organizações inteligentes, sendo o conhecimento um novo fator de produção. Aliás, o conhecimento passa a ser considerado o principal fator de produção, produtividade, agregação de valor e crescimento econômico. Assim, gerenciar o conhecimento e saber como compartilhá-lo tornam-se atividades de extrema importância no contexto organizacional atual (EVERS, 2001).

A gestão do conhecimento é definida porO'Leary (1998) como ogerenciamento do conhecimento com o intuito de facilitar sua criação, possibilitar acessá-lo e reutilizá-lo. Segundo Liebowitz e Wilcox (1997), a gestão do conhecimento consiste na habilidade que as organizações têm para gerenciar conhecimento, armazená-lo, agregar valor e distribuí-lo. Além disso, pode-se inferir que a gestão do conhecimento é o processo de criação, captura e utilização do conhecimento com o objetivo de melhorar o desempenho organizacional (BASSI, 1999).

Autores como Sá et al. (2013, p. 127-128), por sua vez, salientam que a gestão do conhecimento "é uma atividade gerencial, voltada a desenvolver um conjunto de ações com o objetivo de fomentar o conhecimento organizacional". As referidas ações devem estimular tanto a criação quanto a explicitação e disseminação do conhecimento no âmbito organizacional.

Conforme Freire et al. (2012), o desempenho organizacional tende a melhorar em organizações que preservam e gerenciam o conhecimento de seus colaboradores, pois tal comportamento permite à organização repetir experiências que deram certo, adicionar novos conhecimentos e, além disso, evitar erros cometidos anteriormente. A rotatividade em virtude da saída dos indivíduos de uma organização é uma realidade inevitável; assim sendo, as organizações devem buscar gerenciar o conhecimento de seus membros, captando, registrando e disseminando-o. Desse modo, a rotatividade dentro de uma organização pode ser considerada um fator positivo, somando novos conhecimentos aos previamente existentes em determinada organização em vez de o conhecimento sair da organização com o desligamento de determinado profissional do grupo.

Recomenda-se que o conhecimento seja compartilhado cotidianamente no trabalho e, em seguida, codificado e registrado em bancos de dados, estando disponível quando necessário. Quanto mais restrito for o ambiente detentor do conhecimento organizacional, mais vulnerável e fragilizada estará a organização, uma vez que o poder de barganha e de influência dos detentores desse conhecimento será maior. Assim, considerando-se a importância e necessidade do conhecimento organizacional, meios competentes de registro de informações são indispensáveis, principalmente 
no que concerne às informações relevantes, a fim de armazená-las e torná-las acessíveis (FREIRE et al., 2012).

\subsection{Compartilhamento do conhecimento organizacional}

Conforme Freire et al. (2012), o conhecimento deve ser explicitado e codificado (tornando-se conhecimento explícito) para que possa ser armazenado e utilizado por outras pessoas na criação de novos conhecimentos. Uma das etapas mais importantes no processo de gestão do conhecimento consiste em compartilhar o conhecimento armazenado para que outras pessoas e/ou organizações possam usá-lo eficientemente.

De acordo com Argote e Ingram (2000), o compartilhamento de conhecimento nas organizações é um processo no qual determinada unidade é afetada pela experiência de outra. Embora o compartilhamento de conhecimento em organizações envolva compartilhamento no nível individual, o problema do compartilhamento do conhecimento nas organizações pode ocorrer em outros níveis, como grupo, departamento, etc. Rocha e Terra (2008) esclarecem que existem várias maneiras de compartilhar conhecimento e diversos modos para analisar os mecanismos que podem ser utilizados, tais como portais corporativos e Intranet; melhores práticas (best practises); treinamentos e expatriados, entre outros.

Dentre os principais componentes das práticas do conhecimento, Sá et al. (2013, p. 127) destacam fatores relacionados, como, por exemplo, política cultural, "políticas de recursos humanos, estratégia e comunicação organizacional, estratégias do conhecimento, estímulo à aprendizagem, clima organizacional e feedback", como também a mensuração das capacidades e resultados, e acesso à informação.

O elemento criador do conhecimento é o indivíduo, sendo a organização o elemento ampliador e o grupo, o elemento de síntese do conhecimento. Saber compartilhar o conhecimento dentro da empresa, a fim de que ele não fique concentrado em algumas pessoas, apresenta-se como um desafio para os gestores (NONAKA; TAKEUCHI, 1997). Assim, o gerenciamento eficiente do conhecimento nas organizações dependerá, primeiramente, do gerenciamento individual dos elementos que compõem a gestão do conhecimento e, posteriormente, da sinergia entre eles (RAUPP; BEUREN, 2007).

O compartilhamento de conhecimentos científicos e tecnológicos pode ser alavancado por meio de parcerias entre universidades e organizações do segmento empresarial. Esse tipo de parceria é realidade e prática em economias de países como Japão, Estado Unidos, Inglaterra e Alemanha, entre outros. Nesses países, acredita-se que a parceria entre os referidos segmentos pode incrementar a competitividade, bem como o desenvolvimento científico e tecnológico, e a difusão de tecnologias, destacando-se como um dos principais instrumentos de difusão de tecnologias que induzem ao desenvolvimento (PEREIRA et al., 2009).

Além disso, existem diversas formas de compartilhamento do conhecimento e elas são estudas com a finalidade de identificar a(s) mais apropriada(s) para cada caso, garantindo a eficiência do processo. As principais formas de compartilhamento do conhecimento citadas por Raupp e Beuren (2007) são reuniões, encontros, palestras, Intranet, seminários, discussões, livros, revistas, jornais e realização de pesquisas. De acordo com Souza et al. (2008), repositórios de projetos inovadores e bemsucedidos também podem ser considerados ferramentas para compartilhamento do conhecimento organizacional.

Na concepção de Raupp e Beuren (2007), deve haver compartilhamento e troca de conhecimentos entre empreendedores de ramos similares ou mesmo de ramos diferentes, pois podem, em diversas ocasiões, detectar as mesmas dificuldades durante a realização de determinado processo. Assim, analisar não apenas o compartilhamento do conhecimento entre os gestores e empregados ou entre empregados e empreendedores, mas, também (e principalmente), entre empreendedores, pode trazer facilidades e vantagens a todos os envolvidos.

\subsection{Gestão do conhecimento em empresas de base tecnológica}

Em empresas de base tecnológica, há uso constante e intensivo do conhecimento, tanto científico quanto tecnológico, e esta é uma condição essencial para que tais empresas mantenhamse competitivas e materializem conhecimentos. A materialização do conhecimento pode ocorrer por intermédio de inovações tecnológicas oferecidas pelas empresas de base tecnológica ao mercado. As empresas de base tecnológica desempenham um papel importante no desenvolvimento socioeconômico da sociedade, especialmente nas regiões onde atuam. Nesse contexto, podem receber apoio de habitats de inovação, como incubadoras, por exemplo, que podem oferecer estrutura e recursos, estimulando a convergência do conhecimento (TUMELERO et al., 2011). 
Complementando as afirmações de Tumelero et al. (2011), autores como Pinho (2005) e Ferreira, Oliveira Jr. e Borini (2012) ressaltam que empresas de base tecnológica são organizações de setores de alta tecnologia, que concentram seus esforços e operações na criação de novos produtos, e caracterizam-se pela realização de esforços tecnológicos expressivos, podendo estar articulados com universidades e/ou instituições de pesquisa. Ou seja, com o intuito de reforçar vínculos e fortalecer-se no ambiente competitivo, pode haver articulações e parcerias entre empresas de base tecnológica e outras organizações.

Além disso, Ferreira, Oliveira Jr. e Borini (2012) tratam da existência de barreiras na estrutura do ambiente empresarial brasileiro, que dificultam a atuação das empresas de base tecnológica. Como exemplos de barreiras, os autores citam a deficiência existente nas estruturas empresariais, tanto de funções administrativas quanto comerciais, a fragilidade apresentada pelas redes de cooperação e as dificuldades de adequar e certificar os produtos de acordo com as normas internacionais.

A respeito de fatores que podem trazer facilidades às empresas de base tecnológica, Ferreira, Oliveira Jr. e Borini (2012) ressaltam que, quando tais empresas adquirem experiência no mercado nacional (doméstico), passam a contar com pontos favoráveis também para o processo de internacionalização, com fatores como parcerias com institutos de pesquisa, universidades e empresas multinacionais; além de contarem com a possibilidade de utilizar políticas governamentais de apoio.

A possibilidade de compartilhar/trocar conhecimentos e experiências com instituições especializadas pode trazer inúmeros benefícios às organizações, especialmente às empresas de base tecnológica, pois são eminentemente dependentes do conhecimento como fator de produção e agregação de valor. As empresas de base tecnológica podem, igualmente, ser chamadas de empresas de tecnologia de ponta ou empresas de tecnologia avançada (TUMELERO et al., 2011).

\section{PROCEDIMENTOS METODOLÓGICOS}

Para analisar o processo de gestão do conhecimento em uma empresa de base tecnológica, foi realizado um estudo de caso do tipo exploratório e explicativo. Estudo de caso trata-se de uma estratégia de investigação em que o pesquisador explora uma atividade, um processo ou um ou mais indivíduos (CRESWELL, 2010).

A pesquisa é, igualmente, de natureza exploratória, pois visa extrair informações referentes à gestão do conhecimento e suas práticas, adotadas dentro da empresa estudada. E, conforme Lakatos e Marconi (1990), a análise ou explicação é a tentativa de evidenciar as relações existentes entre o fenômeno estudado e outros fatores.

Para conseguir as informações necessárias à pesquisa, foram realizados dois tipos de coleta de dados diferentes com dois colaboradores da empresa. A primeira delas foi feita por meio de um instrumento de pesquisa com perguntas abertas que foram enviadas por e-mail aos dois funcionários simultaneamente; e a segunda, por intermédio de ligações telefônicas para os colaboradores com o objetivo de conhecer melhor a realidade da empresa.

Com as perguntas abertas, tinha-se como objetivo colher informações sobre o sistema de gestão do conhecimento adotado pela empresa pelos dois colaboradores respondentes.

Os dois colaboradores escolhidos foram: um gestor da área de conhecimento da empresa e o outra, uma analista de qualidade de software. Eles foram selecionados pelos seguintes motivos: o gestor do conhecimento por ser o responsável pela área de interesse deste estudo dentro da empresa e o analista de qualidade de software por ter a visão de um colaborador comum sobre a gestão do conhecimento da organização.

As perguntas abertas do instrumento de pesquisa foram formuladas com base na literatura a respeito de implantação da gestão do conhecimento em organizações de base tecnológica.

Em seguida, foi realizada uma comparação entre as respostas dos dois entrevistados para compreender como ocorre o processo de gestão do conhecimento na empresa do ponto de vista do gestor do conhecimento e de uma pessoa que utiliza o conhecimento na empresa (analista de software).

\section{ANÁLISE DOS RESULTADOS}

Nesta seção, serão apresentadas as perguntas e respostas enviadas aos representantes da empresa, objeto desta pesquisa. Como também uma análise do que pode significar as respostas para cada um dos questionamentos. 
As respostas do analista de software são indicadas por AS e as do gestor do conhecimento por GC:

1) Qual a relação da empresa com gestão do conhecimento? Existem práticas internas?

- AS: Sim, existem Workshops, treinamentos gravados, Webniar dos produtos para clientes, funcionários e a sociedade.

- GC: Utilizamos a gestão do conhecimento para os conhecimentos já existentes, para criarmos repositórios de conhecimento e melhorar o acesso ao conhecimento. Temos como práticas internas a criação de procedimentos e treinamentos, repasse de conhecimento, reuniões, treinamentos online ao vivo, e-learning e ferramentas de colaboração.

2) Relate uma ferramenta ou prática interna da empresa quanto à gestão do conhecimento.

- AS: Ferramenta de e-learning utilizada para integração de novos funcionários, bem como aprimoramento da carreira dos profissionais com a possibilidade de utilização de trilha de aprendizagem.

- GC: A capacitação dos novos colaboradores na função da área de pós-entrega é realizada através de uma árvore de conhecimento na qual já temos os treinamentos e recursos estabelecidos. Além disso, já disponibilizamos para os novos colaboradores uma trilha de integração que irá facilitar a gestão do conhecimento; logo pretendemos ter trilha do conhecimento de todos os produtos, multiplicadores do conhecimento, e continuar documentando nossos conhecimentos através de Manual de Referência, Faqs, procedimentos e e-learnings. Recentemente inauguramos o portal do conhecimento que disponibilizará todos os e-learnings, documentos, Trilhas do Conhecimento e ferramentas de colaboração como: fórum, chat e enquete.

3) Quando iniciou essa prática? De que departamento surgiu?

- AS: Quando iniciou não tenho essa informação, a empresa tem 15 anos, provavelmente surgiu junto com a empresa, e teve algumas etapas de evolução. Surgiu da área de Recursos Humanos (RH) e Educação Corporativa.

- GC: Esta prática já existia em algumas áreas, porém a capacitação dos novos na função teve início em 2013 na área de pós-entrega. Outras ações estão em andamento, como a criação das trilhas e programa de multiplicadores do conhecimento.

4) Já tem resultados? Quais?

- AS: Sim, o tempo de "setup" para um novo funcionário é menor, visto que com a ferramenta os treinamentos são on-line, realizados de acordo com a agenda do funcionário novo, ou até mesmo dos funcionários antigos, os quais podem estar reciclando seus conhecimentos.

- GC: Antes o acompanhamento dos novos colaboradores era realizado presencialmente, agora com todos os treinamentos já preestabelecidos na trilha conseguimos aumentar a produtividade.

5) Se não tem resultados ainda, quais os resultados esperados?

- AS: "Respondeu à pergunta quatro que houve resultados".

- GC: Nosso objetivo é ter um repositório com todos os conhecimentos e cada vez mais ter ferramentas de colaboração para facilitar a gestão do conhecimento.

6) O que você acha dessa prática de gestão do conhecimento na empresa?

- AS: Acho prático, ágil e necessária, pois estamos na era digital, onde a reciclagem do conhecimento é fundamental para evolução de nossa carreira, e com a possibilidade de fazermos de acordo com nossa agenda ganhamos praticidade e mais aproveitamento do treinamento.

O Quadro 1 mostra os pontos em comum entre as respostas do analista de software e as do gestor do conhecimento.

Quadro 1 - Pontos em comum das respostas às questões sobre gestão do conhecimento

\begin{tabular}{|c|l|}
\hline Perguntas & \multicolumn{1}{|c|}{ Pontos em comum } \\
\hline 1 & Existem treinamentos, treinamentos on-line, e-learning. \\
\hline 2 & $\begin{array}{l}\text { Capacitação de novos colaboradores, para integração através de e-learning ou trilha da } \\
\text { integração. Para aprimoramento dos profissionais, a trilha de aprendizagem ou trilha do } \\
\text { conhecimento, esta ferramenta está sendo construída. }\end{array}$ \\
\hline 3 & $\begin{array}{l}\text { A prática de GC já existia em algumas áreas; o colaborador AS disse que surgiu na área de RH, } \\
\text { área do Gestor do Conhecimento. }\end{array}$ \\
\hline 4 & Aumento da produtividade dos novos colaboradores. \\
\hline 5 & $\begin{array}{l}\text { Objetivo do GC: ter repositórios do conhecimento. Como disse o AS na resposta quatro, é } \\
\text { importante para reciclar conhecimentos dos antigos colaboradores também. }\end{array}$ \\
\hline 6 & Necessária para reciclagem do conhecimento. \\
\hline
\end{tabular}

Fonte: Elaborado pelos autores (2014) 
De acordo com as respostas obtidas por meio de perguntas enviadas por e-mail e, num momento seguinte, por telefone, visando à coleta de informações complementares, alguns pontos em comum entre as respostas do analista de software e as do gestor do conhecimento foram encontrados.

No que se refere às práticas de gestão do conhecimento utilizadas pela empresa, foi constatado que as principais práticas existentes são treinamentos, treinamentos on-line e e-learning. Em relação à ferramenta ou prática interna da empresa para gestão do conhecimento, foi constatado que e-learning é bastante usado para capacitação e integração de novos colaboradores. Como ferramenta para aprimoramento dos profissionais, está sendo construída a trilha de aprendizagem ou trilha do conhecimento.

$\mathrm{Na}$ empresa estudada, as práticas de gestão do conhecimento já existiam há algum tempo em diversos setores, tendo surgido por iniciativa do setor de $\mathrm{RH}$. Como resultado da aplicação de práticas de gestão do conhecimento, os entrevistados foram unânimes em destacar o aumento da produtividade. A empresa espera ainda criar outras práticas, como, por exemplo, repositórios de conhecimento. Para finalizar, foi respondido que os funcionários estão gostando de adotar tais práticas, sobretudo, como forma de reciclar seus conhecimentos.

\section{CONSIDERAÇÕES FINAIS}

Ponderando as informações apresentadas neste estudo, pode-se inferir que compartilhar conhecimentos pode trazer vantagens às organizações, especialmente àquelas empresas que dependem no seu dia a dia do conhecimento, como é o caso dos empreendimentos de base tecnológica, que são dependentes do conhecimento como fator de produção. Em virtude do uso constante e intensivo do conhecimento na referida empresa, este é considerado essencial para que elas mantenham-se competitivas e possam utilizar do conhecimento de diversas formas para convergir e transformar-se em inovações tecnológicas de produtos e/ou serviços (TUMELERO et al., 2011).

Para realizar o compartilhamento de conhecimento, as empresas de base tecnológica podem fazer uso de diversas ferramentas. No caso específico da empresa analisada neste estudo, as principais ferramentas de gestão do conhecimento identificadas são e-learning, trilha da integração para novos colaboradores e, segundo os entrevistados, futuramente, espera-se ter repositórios de conhecimento.

Porém, conforme apresentado por Ferreira, Oliveira Jr. e Borini (2012), podem existir barreiras tanto no processo de implementação quanto na continuação das atividades de gestão do conhecimento. Os entrevistados no presente estudo, ao serem questionados sobre as barreiras para implantação de gestão do conhecimento, responderam que não foram identificadas barreiras nesse processo, uma vez que todos percebiam a necessidade do repositório e e-learning (os entrevistados acreditam que a facilidade deu-se pelo tipo de mão de obra da empresa). Tal resposta igualmente pode ser considerada como um ponto da empresa que os colaboradores optaram por não revelar na pesquisa.

Com o intuito de reduzir e/ou evitar a existência de barreiras ao processo de gestão do conhecimento, Sá et al. (2013) recomendam que a gestão do conhecimento seja implementada aos poucos, com a participação de todos os funcionários de forma efetiva, para que eles sintam-se parte do processo. Os autores salientam, ainda, que vários aspectos são englobados nesse tipo de gestão e, desse modo, não se trata unicamente da construção do conhecimento, mas também de gerir os processos necessários para desenvolvê-lo, preservá-lo, compartilhá-lo e utilizá-lo, transformando-o em competências, para atingir os objetivos organizacionais.

Ainda no que diz respeito às ferramentas, analisando as respostas e comportamentos apresentados de acordo com a realidade da empresa em questão, observou-se que não foram colocadas as Comunidades de Prática (CoPs) como exemplo. Porém, com base nas respostas da pesquisa, a empresa faz uso da ferramenta scrum para o desenvolvimento de software e entrega de tarefas das áreas. Referida ferramenta possibilita o trabalho de colaboradores de áreas diferentes em um determinado projeto.

Assim, acredita-se que o uso de tal ferramenta igualmente poderia ser aproveitado como meio para gestão do conhecimento da empresa, uma vez que todos os colaboradores têm acesso a ele e estão acostumados a trabalhar em projetos em conjunto, utilizando esses meios.

Ressalta-se que é apenas uma sugestão, pois a empresa pode escolher outras maneiras para implementar as Comunidades de Prática e utilizar outros softwares, até mesmo outra ferramenta conhecida ou desenvolvida pela própria empresa.

Conforme inferido por Sá et al. (2013), mudanças significativas vêm ocorrendo no mundo todo 
e, especialmente, no contexto organizacional. Tais mudanças são causadas pelos fenômenos da competitividade e internacionalização das economias, fazendo com que as empresas abram-se a modelos de gestão centrados no conhecimento. No entanto, os mesmos autores destacam que a gestão do conhecimento não é simples de ser implantada, uma vez que requer o envolvimento de toda a empresa.

$\mathrm{Na}$ análise das respostas dadas pelos dois colaboradores da empresa objeto de estudo desta pesquisa, percebeu-se que há pontos em comum entre eles, como: ambos falaram sobre a importância dos treinamentos on-line para novos colaboradores e da trilha do conhecimento para os profissionais mais antigos. Também expuseram que a gestão do conhecimento já existia na empresa e que um dos resultados seria a produtividades dos novos colaboradores. $O$ repositório igualmente é uma das ferramentas apontadas como de alto grau de importância para a empresa.

Conclui-se, com a realização deste estudo, que a gestão do conhecimento na empresa estudada está em andamento e já apresentou alguns resultados percebidos tanto pelo gestor do conhecimento como por outro colaborador usuário do conhecimento. Nessa fase de desenvolvimento, Sá et al. (2013, p. 127) ressaltam a existência de dificuldades no processo de implantação da gestão do conhecimento, mas reforçam que "seus resultados trazem grandes benefícios competitivos para a organização".

\title{
ANALYSIS OF THE KNOWLEDGE MANAGEMENT PROCESS IN A TECHNOLOGY- BASED COMPANY FROM JOINVILLE-SC
}

\begin{abstract}
Currently knowledge is the main factor of production organizations. Among the companies that stand out as knowledge intensive organizations are the technology based organizations. The objective of this study was to show the actions of knowledge management of a technology-based company from Joinville-SC. A qualitative, exploratory and explanatory case study was carried out, using primary data collected in two steps. The first collection was made through a survey with open questions which was sent by e-mail to two collaborators of the organization concerned. The second through calls to the same collaborators in order to better understand the reality of the business and understand the answers to the questionnaire. Among the main results we can mention the fact that the company makes use of tools such as communities of practice, identified in organizational routines, but these were not mentioned by the respondents.
\end{abstract}

Keywords: Management . Knowledge. Company. Technology. 


\section{REFERÊNCIAS}

ARGOTE, L.; INGRAM, P. Knowledge Transfer: A Basis for Competitive Advantage in Firms.

Organizational Behavior and Human Decision Processes, San Diego, v. 82, n. 1, p. 150-169, 2000.

Disponível em: <http://www.idealibrary.com>. Acesso em: 12 jun. 2014.

BASSI, L. Harnessing the power of intellectual capital. In: CORTADA, J.; WOODS, J. The Knowledge Management Yearbook. Boston: Butterworth-Heinemann, 1999. p. 211-220.

CRESWELL, J.W. Projeto de pesquisa: métodos qualitativo, quantitativo e misto. Porto Alegre: Artmed, 2010.

EVERS, H. D. Towards a Malaysian Knowledge Society. [2008]. Disponível em: <http://www. researchgate.net/profile/Hans-Dieter_Evers/publication/252492284_Transition_towards_a_ Knowledge_Society_Malaysia_and_Indonesia_Compared/links/00b495215f0f $\overline{2} 1704000000$.pdf $>$. Acesso em: 4 jun. 2015.

FERREIRA, F. R.; OLIVEIRA, JR. M. M.; BORINI, F. M. Internacionalização acelerada de empresas de base tecnológica: o caso das born globais brasileiras. Revista de Administração Contemporânea, Rio de Janeiro, v. 16, n. 6, p. 866-888, nov./dez. 2012.

FREIRE, P. de S. et al. Memória organizacional e seu papel na Gestão do conhecimento. RCA Revista de Ciências da Administração, Florianópolis, v. 14, n. 33, p. 41-51, 2012. Disponível em: $<$ https://periodicos.ufsc.br/index.php/adm/article/view/2175-8077.2012v14n33p41/22535>. Acesso em: 21 jun. 2014.

JACQUES, J. É.; GONÇALO, C. R. Gestão estratégica do conhecimento baseada na construção de protocolos médico-assistenciais: o compartilhamento de idéias entre parcerias estratégicas como vantagem competitiva. RAI - Revista de Administração e Inovação, São Paulo, v. 4, n. 1, p. 106124, 2007. Disponível em: <http://www.revistarai.org/rai/article/view/76/87>. Acesso em: 22 jun. 2014.

LAKATOS, E. M.; MARCONI, M. A. Fundamentos de Metodologia Científica. 2. ed. São Paulo: Atlas, 1990.

LIEBOWITZ, J.; WILCOX, L. Knowledge management and its integrative elements. Boca Raton: CRC Press, 1997.

LOBO, M. R. Gestão do conhecimento como diferencial competitivo: uma perspectiva a partir do capital humano. [2010]. Disponível em: <http://www.excelenciaemgestao.org/Portals/2/ documents/cneg6/anais/T10_0326_1115.pdf>. Acesso em: 26 jun. 2014.

NONAKA, I.; TAKEUCHI, H. Criação de conhecimento na empresa: como as empresas japonesas geram a dinâmica da inovação. Rio de Janeiro: Campus, 1997.

O`LEARY, Z. The essential guide to doing research. Londres: Sage Publications, 1998.

PEREIRA, M. F. et al. Transferência de conhecimentos científicos e tecnológicos da universidade para o segmento empresarial. RAI - Revista de Administração e Inovação, São Paulo, v. 6, n. 3, p. 128 144, 2009. Disponível em: <http://www.revistarai.org/rai/article/view/406/246>. Acesso em: 22 jun. 2014.

PINHO, M. Empresas de base tecnológica: diretório da pesquisa privada. São Carlos-SP: Universidade Federal de São Carlos, 2005.

RAUPP, F. M.; BEUREN, I. M. Compartilhamento do conhecimento em incubadoras brasileiras associadas à ANPROTEC. RAM - Revista de Administração Mackenzie, São Paulo, v. 8, n. 3, p. 38-58, 2007. Disponível em: <http://editorarevistas.mackenzie.br/index.php/RAM/article/ view/123/123>. Acesso em: 12 maio 2014.

ROCHA, T.; TERRA, J. C. A Transferência de Conhecimento em Marketing em Multinacionais: Estudo em Subsidiárias Brasileiras. In: Encontro da ANPAD, 32., 2008. Anais eletrônicos..., Rio de Janeiro: ANPAD, 2008. p. 1-16. 
SÁ, F. B. et al. Práticas de gestão do conhecimento: um estudo em organizações mineiras. Perspectivas em Gestão \& Conhecimento, João Pessoa, v. 3, n. 1, p. 114-131, jan./jun. 2013. Disponível em: <http://periodicos.ufpb.br/ojs/index.php/pgc/article/view/12259/9326>. Acesso em: 26 nov. 2014.

SANTOS, D. T. dos; PINHO M. Análise do crescimento das empresas de base tecnológica no Brasil. Produção, São Carlos, v. 20, n. 2, p. 214-223, 2010. Disponível em: <http://www.scielo.br/pdf/prod/ v20n2/aop_200806057.pdf >. Acesso em: 26 jun. 2014.

SOUZA, C. A. de et al. Portais de disseminação de práticas em tecnologias de informação e comunicação no setor público: o caso do Observe-gov. RAUSP-e - Revista de Administração - eletrônica, São Paulo, v. 1, n. 2, p. 1-24, 2008. Disponível em: <http://www.rausp.usp.br/Revista_ eletronica/v1n2/artigos/resumo_v1n2a8.htm>. Acesso em: 12 jun. 2014.

TUMELERO, C. et al. Estudo do Conhecimento em Empresas de Base Tecnológica Incubadas: Proposição de um Modelo Conceitual Integrativo. Revista Gestão \& Tecnologia, Pedro Leopoldo, v. 11, n. 1, p. 2-17, jan./jun. 2011.

URIARTE JR., Filemon A. Introduction to Knowledge Management. Jakarta Indonesia: ASEAN Foundation, 2008. 